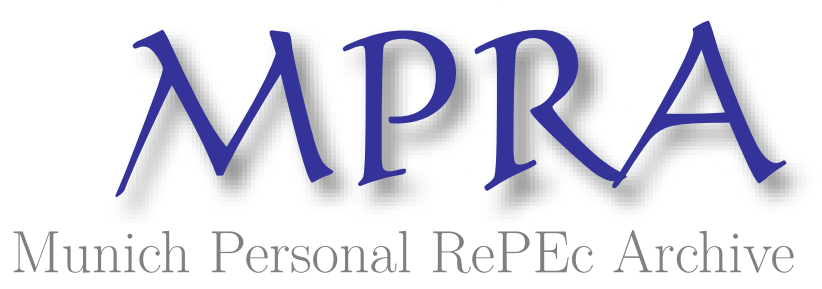

\title{
Stochastic Specification and Maximum-Likelihood Estimation of the Linear Expenditure System
}

Chipman, John S. and Tian, Guoqiang

1989

Online at https://mpra.ub.uni-muenchen.de/41385/

MPRA Paper No. 41385, posted 17 Sep 2012 13:35 UTC 


\section{STOCHASTIC SPECIFICATION AND MAXIMUM-LIKELIHOOD ESTIMATION OF THE LINEAR EXPENDITURE SYSTEM*}

\author{
JOHN S. CHIPMAN \\ University of Minnesota \\ Department of Economics \\ Minneapolis, MN 55455 \\ U.S.A.
}

\author{
GUOQIANG TIAN \\ Texas A \& M University \\ Department of Economics \\ College Station, TX 77843-4228 \\ U.S.A.
}

ABSTRACT. From the point of view of consumer demand theory the linear expenditure system (LES) provides a convenient model for representing consumer response to price and income and its linearity is one of its most attractive features. But when estimation problems are discussed, the descriptive adjective is more notable for its irony than its accuracy. Since Stone (1954) first calculated parameter estimates for the LES, some stochastic specifications for the system have been given. These specifications, however, ignore some of the requirements implied by economic theory and these methods of estimation lack desirable properties. This paper will deal with the problems of stochastic specification and maximum-likelihood estimation of the IES making full use of the restrictions of economic theory by assuming that the minimum required quantities for the commodities have a three-pacter distribution.

\section{Introduction}

The linear expenditure system (LES) - first introduced by Klein and Rubin (1948) - has had great appeal in applied econometrics owing to the fact that it expresses consumer expenditures on the various commodities as linear functions of prices and income, consistently with the hypothesis of utility-maximization subject to a budget constraint. It was shown by Samuelson (1948) (and subsequently again by Geary, 1949) that the corresponding system of demand functions is generated by a utility function which is linear in the logarithms of displacements of the quantities consumed, or equivalently, by a shifted Cobb-Douglas utility function. Stone (1954) was the first to apply this concept empirically, using an ingenious heuristic computational algorithm. This was further developed in Stone (1964) and Stone et al. (1964), and the approach has been the subject of a valuable exposition and development by Deaton (1975).

The pure theory of consumer behavior makes no allowance for the random errors that must be allowed for in statistical estimation. The procedure followed by Stone's followers, such as Barten $(1964,1969)$, Theil $(1965)$, Malinvaud (1966), Parks $(1969,1971)$, Deaton (1975) and others has been to add random terms to the expenditure functions, without considering (as in the original deterministic formulation of Klein and Rubin, 1948) whether the new stochastic demand functions could find a basis in the theory of rational behavior, except in the following limited sense: it was noted independently by Barten $(1969)$ and Parks $(1969,1971)$ that if the stochastic demand functions were to obey the budget constraint exactly, then the covariance matrix of the error terms would necessarily be singular, and that this restriction should be taken into account in 
the development of efficient estimation procedures. However, this still leaves unanswered the question of the source of the error terms. If it is not a random error in the consumer's computation of the budget constraint it must be a random error in the consumer's utility function.

The idea that preferences themselves are stochastic was apparently first suggested by Mosteller and Nogee (1951). They noted that in experiments to test transitivity of preferences, one could always find instances in which this postulate was violated unless it were recast in stochastic form. A large literature on stochastic choice emerged, of which one may cite May (1954), Quandt (1956), Papandreou (1957), Luce (1958), Debreu (1958), Davidson and Marschak (1959), Chipman (1960), and Block and Marschak (1960). The basic idea is that an individual's preference ordering is conditional on a particular 'state of mind' which can change from time to time in response to intemal or external stimuli. This approach has been generally accepted in psychology but has been slow to penetrate econometrics, except in the area of discrete-choice models, where it has very successfully been applied by McFadden (1974). In the continuouschoice setting of the theory of consumer demand the only applications we are aware of are those of Pollak and Wales (1969) and Woodland (1979), and more recently Lee and Pitt (1986a, 1987, 1986b). Pollak and Wales (1969) included a random error term in the Klein-Rubin Geary utility function, but unfortunately they postulated it to have a normal distribution, which is logically impossible since the logarithm is defined only for positive numbers. Woodland (1979) suggested an alternative specification on the disturbances in the LES, namely that they have a Dirichlet distribution. However, the Dirichlet distribution requires the expenditures for the different commodities to be independent of one another, by the definition of the Dirichlet distribution (see Rao 1973, p. 125). This is clearly a very strong assumption.

The main purpose of this paper is to remove those obstacles and suggest an alternative stochastic specification. We assume that the stochastic term in the utility function has a multivariate lognormal distribution and then obtain the first-order conditions from which the maximum-likelihood (ML) estimators are derived. It will be noted that our approach satisfies all of the restrictions implied by economic theory in contrast to some more general approaches such as that of the 'Rotterdam model' (Barten 1964, 1969; Theil 1965) - which uses a quadratic approximation to the utility function - and the model of Christensen, Jorgenson, and Lau (1975) - which uses a utility function which likewise does not satisfy the global concavity and monotonicity constraints assumed in consumer theory. Though this paper considers the estimation problem only for the case of the LES model, we think it quite likely that some of the techniques developed in the paper can be profitably used in other expenditure-share models, e.g., the Rotterdam differential demand model, the Christensen-Jorgenson-Lau model, and the Deaton and Muellbauer (1980b) AIDS model. Our aim in this paper is not to strive for the utmost generality but to try to carry out an exact analysis for the simplest case in the the utmost methods can later be applied to more general cases.

One of the noteworthy consequences of the stochastic formulation undertaken in this paper is that the error terms in the expenditure equations depend upon the prices, see equation (5) below; thus, the usual procedures - if our formulation is correct - fail to take account of the pronounced heteroskedasticity in the error term of the LES that could result from price fluctuations.

One limitation of the formulation of this paper should be mentioned: no account is taken of boundary conditions. That is, it is assumed that the first-order conditions of an interior maximum are satisfied for the consumer. For most applications, where commodity aggregation assures that there will be positive consumption in each category, this should present no problem; however, this might no longer be so in the case of highly disaggregated data sets. For treatments of these problems see Wales and Woodland (1983) and Lee and Pitt (1986a, 1987, 1986b).

The plan of the paper is as follows: Section 2 begins with the discussion of the stochastic specification by making systematic use of economic theory. Section 3 gives the first-order conditions of the ML estimator for the LES under the lognormal specification. In Section 4 we report our conclusions.

\section{Stochastic Specification}

Let $q_{t j}$ be the quantity of commodity $j$ demanded at time $t(j=1,2, \ldots, m ; t=1,2, \ldots, T)$, and let it be assumed that the community acts as if it maximized a stochastic utility function

$$
U\left(q_{i} ; \alpha, \gamma_{t}, \varepsilon_{i}\right)=\sum_{i=1}^{m} \alpha_{i} \log \left(q_{i j}+\gamma_{i j}+\varepsilon_{i}\right)\left(\alpha_{i}>0, \sum_{i=1}^{m} \alpha_{i}=1\right)
$$

subject to the budget constraint

$$
\sum_{i=1}^{m} p_{i} q_{i i} \leqq c_{i}
$$

where $p_{t}=\left(p_{t 1}, p_{t 2}, \ldots, p_{t m}\right)^{\prime}$ is the price vector with $p_{t i}>0(i=1,2, \ldots, m)$ at time $t, c_{t}$ is income, or more properly expenditure, at time $t$. The term $\varepsilon_{t}=\left(\varepsilon_{t 1}, \ldots, \varepsilon_{t m}\right)^{\prime}$ is a random error that allows for random variation in the minimum requirement; since (1) is undefined for $q_{i i}+\gamma_{i i}+\varepsilon_{i i} \leq 0, \varepsilon_{t}$ must be assumed to be bounded from below (and thus we cannot assume that $\varepsilon_{t}$ has a multivariate normal distribution). We may take this lower bound to be zero, since any departure from zero may be considered to be absorbed in the term $\gamma_{t}=\left(\gamma_{11}, \gamma_{12}, \ldots, \gamma_{t m}\right)^{\prime}$. In particular, $\varepsilon_{t}$ may be postulated to have a two-parameter lognormal distribution with 0 as lower bound; equivalently, the random vector $\gamma_{t}+\varepsilon_{t}$ may be postulated to have a three-parameter multivariate lognormal distribution with the (unknown) parameter $-\gamma_{t}$ as lower bound. The $\gamma_{t}$ parameter is notoriously difficult to estimate (cf. Johnson and Kotz, 1970; Cohen, 1951; Hill, 1963). However, owing to the nature of the LES we can show, as in Chipman and Tian (1988), that the function $h_{t}$ defined by (11) below - which is a function of the parameters to be estimated - is bounded by probability integral functions (Lemma 1 of Chipman and Tian, 1988), and further we can show that the ML estimation of the LES with lognormal distribution is strongly consistent, asymptotically normal, and asymptotically efficient by using the theory of $M$-estimation recently developed by Huber (1967), Duncan (1987), and others.

The $\gamma_{t}$ here are assumed to be of the form

$$
\gamma_{t}=\sum_{i=0}^{n} \Phi_{l}(t) b_{l}
$$

where $\Phi_{0}=I$ is an identity matrix (thus $\left.b_{0}=\gamma_{0}\right), b_{l}=\left(b_{11}, b_{l 2}, \ldots, b_{l m}\right)^{\prime}$ is an $m$-component vector, and $\Phi_{1}(t)=\operatorname{diag}\left(\phi_{11}(t), \phi_{12}(t), \ldots, \phi_{b_{m}}(t)\right)$ is a diagonal matrix $(l=1, \ldots, n)$.

(3) is a very general form for $\gamma_{k}$. A simple special case is that of the linear time trend $\gamma_{t}=\gamma_{0}+b t$,

which was used by Stone, Brown, and Rowe (1964, p. 205). 
Pollak's (1970) dynamic specification for $\gamma_{t}$, which is also a special case of (3), is

$$
\gamma_{t}=\gamma_{0}+Q_{t-1} b \text {, }
$$

which is called linear habit formation, as it takes into account changes in tastes. This specification was already anticipated by Stone $\left(1954\right.$, p. 522). Here $Q_{1}=\operatorname{diag}\left(q_{11}, q_{12}, \ldots, q_{t m}\right)$.

Chipman (1985) in his empirical research on net-import demand functions for the Federal Republic of Germany using monthly German data gives another dynamic specification, one which lakes both seasonality and time trend into account:

$$
\gamma_{t}=\gamma_{0}+a \cos \left(\frac{\pi}{6} t\right)+b \sin \left(\frac{\pi}{6} t\right)+c t
$$

This is also a special case of (3).

There are two interpretations for $\boldsymbol{\gamma}_{i}$ : one is that $-\boldsymbol{\gamma}_{t}$ is interpreted as minimum-required quantities or subsistence; with this interpretation one must have $q_{i} \geq 0$. The other is that $-\gamma_{t}$ is interpreted as the vector of initial endowments of a set of consumers in an open economy, and that $q_{t i}=z_{i j}-\gamma_{i i}$, where $z_{i i}$ is the gross consumption of commodity $i$ on the part of that economy. Accordingly, only $z_{i i}$ is required to be nonnegative; a positive $q_{i j}$ denotes imports and a negative $q_{i i}$ denotes exports of commodity $i$. In this interpretation, $c_{t}$ must be interpreted not as expenditure but as the excess of expenditure over income, or the deficit in the open economy's balance of payments on current account.

Solving the maximization problem (1) with respect to (2), we obtain the linear expenditure equations

$$
p_{t j} q_{i j}=c_{t} \alpha_{j}+\sum_{i=1}^{m} p_{i j} \gamma_{i i}\left(\alpha_{j}-\delta_{i j}\right)+u_{i j},
$$

where $\delta_{i j}$ is the Kronecker delta and $u_{i j}$ is given by

$$
u_{i j}=\sum_{i=1}^{m} p_{i j}\left(\alpha_{j}-\delta_{i j}\right) \varepsilon_{i i} \text {. }
$$

In vector form (4) becomes

$$
w_{t}=c_{t} \alpha+A_{t} \gamma_{t}+u_{t},
$$

where

$$
\begin{aligned}
& w_{t}=P_{t} q_{t} ; \\
& u_{t}=A_{t} \varepsilon_{i} ; \\
& A_{t}=\left(\propto \alpha^{\prime}-I\right) P_{t},
\end{aligned}
$$

and where $\alpha=\left(\alpha_{1}, \alpha_{2}, \ldots, \alpha_{m}\right)^{\prime}, P_{1}=\operatorname{diag}\left(p_{t 1}, p_{t 2}, \ldots, p_{t m}\right)$ and $\mathrm{t}$ is a column vector with ones in all components.

As Parks $(1969,1971)$ pointed out, Stone (1954) and Malinvaud (1966) overlooked the fact that $u_{f}^{\prime} i=0$ and assumed that the covariance matrix of $u_{t}$ is nonsingular. This is clearly not correct. Also, Pollak and Wales (1969) assumed that $\varepsilon_{q}$ has a multivariate normal distribution. However, this specification violates the condition $q_{i}+\gamma_{i}+\varepsilon_{i j}>0$.

To extricate oneself from this sination one has to formulate a suitable stochastic specification for $\varepsilon_{1}$. To do so we assume that one of the minimum-required quantities, say $\gamma_{m}$, is deterministic (i.e., we assume that $\left.\varepsilon_{m}=0\right)$ and that $\tilde{\varepsilon}_{t}=\left(\varepsilon_{11}, \ldots, \varepsilon_{L, m-1}\right)$ has a two-parameter multivariate lognormal distribution with 0 as lower bound. With this specification, the implied stochastic expenditure equations are of the form (4) with

$$
u_{i j}=\sum_{i=1}^{m-1} p_{i i}\left(\alpha_{j}-\delta_{i j}\right) \varepsilon_{i j}
$$

for $j=1,2, \ldots, m$. Thus (4) can be written as

$$
p_{i j} q_{i j}=c_{t} \alpha_{j}+\sum_{i=1}^{m} p_{i j} \gamma_{i}\left(\alpha_{j}-\delta_{i j}\right)+\sum_{i=1}^{m-1} p_{i j}\left(\alpha_{j}-\delta_{i j}\right) \varepsilon_{i},
$$

which are symmetric for all parameters to be estimated. Thus the estimation of the parameters of the LES by (6) will not be affected by which commodity is chosen to be deleted. That is, the

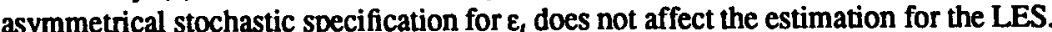

Notice that for any stochastic specification for $\varepsilon_{t}$, the mapping from $\varepsilon_{t}$ to $u_{t}$ is singular (because of $\iota^{\prime} u_{t}=0$ ) and thus the probability density function of $u_{t}$ cannot be expressed explicitly. However, since any expenditure equation of the LES is equal to total expenditure minus the sum of the other expenditure equations, one of the equations (4) is completely redundant in the sense that using the information contained in any $(m-1)$ of the equations we can obtain the $m$-th equation. ${ }^{1}$ Thus we may as well omit the $m$-th equation. The system with the $m$-th equation deleted becomes:

$$
\tilde{w}_{t}=c_{t} \tilde{\alpha}+\tilde{A} \tilde{P}_{t} \tilde{\gamma}_{t}+p_{t m} \gamma_{t m} \tilde{\alpha}+\tilde{u}_{l},
$$

where

$$
\begin{aligned}
& \tilde{w}=\tilde{P}_{1} \tilde{q}_{i} ; \\
& \tilde{A}=\left(\tilde{\alpha} \mathfrak{u}^{\prime}-I\right) ; \\
& \tilde{u}_{t}=\tilde{A}_{t} \tilde{\varepsilon}_{i} ; \\
& \tilde{A}_{t}=\tilde{A} \tilde{P}_{t},
\end{aligned}
$$

and where vectors $\tilde{\alpha}=\left(\alpha_{1}, \alpha_{2}, \ldots, \alpha_{m-1}\right)^{\prime}, \tilde{q}_{t}=\left(q_{t 1}, \ldots, q_{t, m-1}\right)^{\prime}, \tilde{\gamma}_{t}=\left(\gamma_{t 1}, \ldots, \gamma_{t, m-1}\right)^{\prime}$, and matrix $\tilde{P}_{l}=\operatorname{diag}\left(p_{t 1}, p_{t 2}, \ldots, p_{t, m-1}\right)$

Since

$$
\begin{aligned}
\operatorname{det} \tilde{A} & =\operatorname{det}\left[\tilde{\left.\alpha^{\prime}-I\right]}\right. \\
& =\left|\begin{array}{cccc}
\alpha_{1}-1 & \alpha_{1} & \cdots & \alpha_{1} \\
\alpha_{2} & \alpha_{2}-1 & \cdots & \alpha_{2} \\
\cdots & \cdots & \cdots & \cdots \\
\alpha_{m-1} & \alpha_{m-1} & \cdots & \alpha_{m-1}-1
\end{array}\right| \\
& =-\alpha_{m}\left|\begin{array}{cccc}
1 & 1 & \cdots & 1 \\
\alpha_{2} & \alpha_{2}-1 & \cdots & \alpha_{2} \\
\cdots & \cdots & \cdots & \cdots \\
\alpha_{m-1} & \alpha_{m-1} & \cdots & \alpha_{m-1}-1
\end{array}\right| \\
& =(-1)^{m-1} \alpha_{m} \neq 0,
\end{aligned}
$$


we know that the linear transformation

$$
\tilde{u}_{t}=\tilde{\Lambda}, \tilde{\varepsilon}_{t}
$$

is nonsingular and thus we can obtain the distribution of $\tilde{u}_{t}$ from that of $\tilde{\varepsilon}_{i}$.

The assumption that $\tilde{\varepsilon}_{t}$ has a two-parameter multivariate lognormal distribution with 0 as lower bound implies that $x_{t} \equiv \log \tilde{\varepsilon}_{1}$ has a multivariate normal distribution with the mean $a_{t}$ and the covariance matrix $V_{t}$, where $\log \tilde{\varepsilon}_{t}=\left(\log \varepsilon_{t 1}, \ldots, \log \varepsilon_{t, m-1}\right)$. Thus the density function of $\tilde{\varepsilon}_{t}$ is of the form

$$
f\left(\tilde{\varepsilon}_{t}\right)=\frac{\prod_{i=1}^{m-1} \varepsilon_{i i}^{-1}}{(2 \pi)^{\frac{m-1}{2}}\left|V_{t}\right|^{1 / 2}} \exp \left(\xi_{2}\left[\log \tilde{\varepsilon}_{t}-a_{t}\right]^{\prime} V_{t}^{-1}\left[\log \tilde{\varepsilon}_{t}-a_{t}\right]\right\} .
$$

Note that the Jacobian matrix of the transformation from $\tilde{\varepsilon}_{\mathrm{t}}$ to $\tilde{u}_{\mathrm{t}}$ is

$$
\frac{\partial \tilde{\varepsilon}_{t}}{\partial \tilde{u}_{t}}=\tilde{A}_{t}^{-1}
$$

and the absolute value $\left|\operatorname{det} \tilde{\Lambda}_{t}^{-1}\right|=\alpha_{m}^{-1} \prod_{i=1}^{m-1} p_{i i}^{-1}$. To find the density function of $\tilde{w}_{t}$, we use the formula $\tilde{A}^{-1}=-\left(I+\tilde{\alpha} I^{\prime} / \alpha_{m}\right)$ and the facts that

$$
\tilde{A}^{-1} \tilde{\boldsymbol{\alpha}}=-\frac{\tilde{\alpha}}{\alpha_{m}}
$$

and

$$
\tilde{A}^{-1} \tilde{w}_{t}=-\tilde{w}_{t}-\frac{1}{\alpha_{m}} \sum_{i=1}^{m-1} p_{t i} q_{t i} \tilde{\alpha}
$$

Thus by using (7), (8), and $\tilde{A}_{t}^{-1}=\tilde{P}^{-1} \tilde{A}^{-1}$, we have

$$
\tilde{A}_{t}^{-1}\left(\tilde{w}_{t}-c_{t} \tilde{\alpha}-\tilde{A} \tilde{P}_{t} \tilde{\gamma}_{t}-p_{t m} \gamma_{l m} \tilde{\alpha}\right)=\frac{p_{l m}\left(q_{t m}+\gamma_{l m}\right)}{\alpha_{m}} \tilde{P}_{t}^{-1} \tilde{\alpha}-\tilde{q}_{t}-\tilde{\gamma}_{t}
$$

Therefore the probability density of $\tilde{w}_{t}$ is

$$
\begin{aligned}
& h\left(\tilde{w}_{t}\right)=\alpha_{m}^{-1} \prod_{i=1}^{m-1} p_{t i}^{-1} \int\left[\tilde{A}_{t}^{-1}\left(\tilde{w}_{t}-c_{l} \tilde{\alpha}-\tilde{A} \tilde{P}_{t} \tilde{\gamma}_{l}-p_{t m} \gamma_{l m} \tilde{\alpha}\right)\right] \\
& =\alpha_{m}^{-1} \prod_{i=1}^{m-1} p_{i i}^{-1} \frac{\prod_{i=1}^{m-1}\left(\frac{p_{l m}\left(q_{l m}+\gamma_{l m}\right)}{\alpha_{m}} p_{t i}^{-1} \alpha_{i}-q_{i i}-\gamma_{i i}\right)^{-1}}{(2 \pi)^{(m-1) / 2}\left|V_{i}^{1 / 2}\right|} \times \\
& \times \exp \left(-1 / 2\left[\log \left(\frac{p_{l m}\left(q_{m m}+\gamma_{l m}\right)}{\alpha_{m}} \tilde{P}_{t}^{-1} \tilde{\alpha}-\tilde{q}_{t}-\tilde{\gamma}_{t}\right)-a_{t}\right]^{\prime} v_{t}^{-1} \times\right. \\
& \left.\times\left[\log \left(\frac{p_{l m}\left(q_{t m}+\gamma_{m m}\right)}{\alpha_{m}} \tilde{p}_{t}^{-1}-\tilde{\alpha}-\tilde{q}_{t}-\tilde{\gamma}_{t}\right)-a_{t}\right]\right] .
\end{aligned}
$$

\section{Maximum-Likelihood Estimation}

In this section we derive the ML estimator for the parameters to be estimated. For simplicity we assume that the sample observations come from the same population distribution. Distributions for different observations are assumed to be uncorrelated. Thus the logarithm of the likelihood function (9) is

$$
\begin{aligned}
L= & -T \log \alpha_{m}-\sum_{i=1}^{T} \sum_{i=1}^{m-1} \log p_{t i}-\sum_{i=1}^{T} \sum_{i=1}^{m-1} \log h_{t i}-\frac{(m-1) T}{2} \log (2 \pi)- \\
& -\frac{T}{2} \log |\operatorname{det} V|-\frac{1}{2} \sum_{i=1}^{T}\left[\log h_{t}-a\right]^{\prime} V^{-1}\left[\log h_{t}-a\right] .
\end{aligned}
$$

Here

$$
h_{t}=\frac{p_{l m}\left(q_{\iota m}+\gamma_{l m}\right)}{\alpha_{m}} \tilde{P}_{t}^{-1} \tilde{\alpha}-\tilde{q}_{t}-\tilde{\gamma}_{t}
$$

We will obtain the first-order derivatives of $L$ with respect to $a, v, \tilde{\alpha}, \gamma_{0}, b_{l}(l=1, \ldots, n)$. Differentiating (10) with respect to $a$, we have

$$
\frac{\partial L}{\partial a}=\sum_{i=1}^{T} V^{-1}\left[\log h_{i}-a\right]
$$

Letting $\frac{\partial L}{\partial a}=0$, we have

$$
\hat{a}=\frac{1}{T} \sum_{t=1}^{T} \log h_{t} .
$$

Differentiating (10) with respect to $v_{i j}$, we have

$$
\frac{\partial L}{\partial \nu_{i j}}=-e_{i}^{\prime}\left[T V^{-1}-\sum_{i=1}^{T} V^{-1}\left[\log h_{i}-a\right]\left[\log h_{t}-a\right]^{\prime} V^{-1}\right\} e_{j}
$$

for $i \neq j$ and

$$
\frac{\partial L}{\partial v_{i i}}=-\frac{1}{2} e_{i}^{\prime}\left[T V^{-1}-\sum_{i=1}^{T} V^{-1}\left[\log h_{t}-a\right]\left[\log h_{t}-a\right]^{\prime} V^{-1}\right] e_{i},
$$

where $e_{i}$ is an $(m-1) \times 1$ column vector with 1 in the $i$ th place and 0 in other places, and $v_{i j}$ is the element in the $i$ th row and $j$ th column of $V$.

$$
\text { Letting } \begin{aligned}
\frac{\partial L}{\partial V} & =0 \text {, we have } \\
\qquad \hat{V} & =\frac{1}{T} \sum_{t=1}^{T}\left[\log h_{t}-a\right]\left[\log h_{t}-a\right]^{\prime} .
\end{aligned}
$$


Similarly, we can obtain the derivatives of $L$ with respect to $\alpha$ (noting that $\alpha_{m}=1-\sum_{i=1}^{m-1} \alpha_{i}$ ), $\gamma_{0}$ and $b_{l}(l=1,2, \ldots, n)$ :

$$
\begin{aligned}
& \frac{\partial L}{\partial \tilde{\alpha}}=\frac{T}{\alpha_{m}} l-\sum_{t=1}^{T} \frac{p_{\mathrm{tm}}\left(q_{\mathrm{m}}+\gamma_{\mathrm{l} m}\right)}{\alpha_{m}} H_{t}^{-1} \tilde{P}_{t}^{-1} \mathrm{l}- \\
& -\sum_{i=1}^{T} p_{t m}\left(q_{t m}+\gamma_{t m}\right)\left(\alpha_{m}^{-1} I+\alpha_{m}^{-2} \hat{\tilde{\alpha}}\right) H_{i}^{-1} \tilde{P}_{t}^{-1} V^{-1}\left[\log h_{t}-a\right]-\sum_{i=1}^{T} \sum_{j=1}^{m-1} \frac{p_{t m}\left(q_{m m}+\gamma_{t m}\right) \alpha_{j}}{\alpha_{m}^{2} h_{t j} p_{t j}} \iota ; \\
& \frac{\partial L}{\partial \tilde{\gamma}_{0}}=\sum_{t=1}^{T} H_{t}^{-1}\left(t+V^{-1}\left[\log h_{t}-a\right]\right) \\
& \frac{\partial L}{\partial b_{l}}=\sum_{l=1}^{T} H_{l}^{-1} \Phi_{l}(t)\left(l+V^{-1}\left[\log h_{t}-a\right]\right) ; \\
& \frac{\partial L}{\partial \gamma_{0 m}}=-\sum_{t=1}^{T} \sum_{j=1}^{m-1} \frac{p_{t m} \alpha_{j}}{\alpha_{m} h_{t j} p_{t j}}-\sum_{t=1}^{T} \frac{p_{t m}}{\alpha_{m}} \tilde{\alpha}^{\prime} H_{t}^{-1} \tilde{P}_{t}^{-1} V^{-1}\left[\log h_{t}-a\right] ; \\
& \frac{\partial L}{\partial b_{k m}}=-\sum_{t=1}^{T} \sum_{j=1}^{m-1} \frac{p_{l m} \phi_{k m}(t) \alpha_{j}}{\alpha_{m} h_{t j} p_{t j}}-\sum_{t=1}^{T} \frac{p_{l m} \phi_{k m}(t)}{\alpha_{m}} \tilde{\alpha}^{\prime} H_{t}^{-1} \tilde{P}_{t}^{-1} V^{-1}\left[\log h_{t}-a\right],
\end{aligned}
$$

where $H_{t}^{-1}=\operatorname{diag}\left(h_{t 1}^{-1}, \ldots, h_{t, m-1}^{-1}\right), \hat{\tilde{\alpha}}=\operatorname{diag}\left(\alpha_{1}, \ldots, \alpha_{m-1}\right)$, and $\Phi_{t}=\operatorname{diag}\left(\phi_{t 1}, \ldots, \phi_{1, m-1}\right)$. Note that $\gamma_{0}=\left(\tilde{\gamma}_{0}, \gamma_{0 m}\right)^{\prime}$ and $b_{l}=\left(\tilde{b}_{l}^{\prime}, b_{l m}\right)^{\prime}, l=1, \ldots, n$.

The maximum-likelihood estimators are thus obtained by solving the following equations:

$$
\begin{aligned}
\hat{a} & =\frac{1}{T} \sum_{l=1}^{T} \log h_{i} ; \\
\hat{V} & =\frac{1}{T} \sum_{i=1}^{T}\left[\log h_{t}-a\right]\left[\log h_{l}-a\right]^{\prime} ; \\
\frac{\partial L}{\partial \tilde{\alpha}} & =0 ; \\
\frac{\partial L}{\partial \gamma_{0}} & =0 ; \\
\frac{\partial L}{\partial b_{l}} & =0 \text { for } l=1,2, \ldots, n ; \\
\alpha_{m} & =1-\tilde{\alpha}^{\prime} l .
\end{aligned}
$$

Note that the system of equations to be solved is nonlinear in the parameters and we have to resort to numerical methods for solutions. The consistency, asymptotic normality, and efficiency of the ML estimator can be proved in the same way as given in Chipman and Tian (1988).

\section{Conclusion}

This paper has discussed the specification and estimation of the linear expenditure system. It has argued that neither the normal distribution nor the Dirichlet distribution as a specification of the LES can be strictly valid for various reasons associated with the fact that the former does not allow the utility function of the LES to be well-defined while the latter requires that the $w_{t i}$ be mutually independent. To solve the problem we deal with the problems of stochastic specification and maximum-likelihood estimation of the LES making full use of the restrictions of economic theory by assuming that the minimum required quantities for the commodities have a threeparameter multivariate lognormal distribution.

\section{Notes}

* Work done for this paper was supported by the Stiftung Volkswagenwerk, the Riksbankens Jubileum anonymous referee of this volume for useful comments.

1. This is true if the $u_{t}$ are serially independent (see Barten, 1969). However, when the $u_{t}$ are autocorrelated, maximum-likelihood estimates may be conditional on the equation deleted. For a detailed argument, see Berndt and Savin (1975). 


\section{References}

Barten, A. B.: 1964, 'Consumer Demand Functions Under Conditions of Almost Additive Preferences', Econometrica, 32, 1-38.

Barten, A. B.: 1969, 'Maximum-Likelihood Estimation of a Complete System of Demand Equations', European Economic Review, 7-73.

Bemdt, E. R., and N. E. Savin: 1975, 'Estimation and Hypothesis Testing in Singular Equation Systems with Autoregressive Disturbances,' Econometrica, 43, 937-957.

Block, H. D., and J. Marschak: 1960, 'Random Orderings and Stochastic Theories of Response', in I. Olkin et al. (eds.), Contributions to Probability and Statistics, Stanford University Press, Stanford, California, U.S.A.,pp. 97-132.

Chipman, J. S.: 1960, 'Stochastic Choice and Subjective Probability', in D. Willner (ed.), Decisions, Values, and Groups, 1, Pergamon Press, New York, New York, U.S.A., pp. 70-95.

Chipman. J. S.: 1985, 'Estimation of Net-Import Demand Functions for the Federal Republic of Germany, 1959--1982', in H. Giersch (cd.), Probleme und Perspektiven der Weltwirtschaftlichen Entwicklung, Duncker \& Humblot, Berlin, Germany, pp. 197-213.

Chipman, J. S., and G. Tian: 1988, 'Generalized Maximum-Likelihood Estimation of the Linear Expenditure System with Lognormal Distribution', manuscript.

Christensen, L. R., D. W. Jorgenson, and L. J. Lau: 1975, 'Transcendental Logarithmic Utility Functions', American Economic Review, 65, 367-383.

Cohen, A. C.: 1951, 'Estimating Parameters of Logarithmic-Normal Distributions by Maximum Likelihood', Journal of the American Statistical Association, 46, 206-212.

Davidson, D., and J. Marschak: 1959, 'Experimental Tests of a Stochastic Decision Theory', in C. W. Churchman and P. Ratoosh (eds.), Measurement: Definitions and Theories, John Wiley \& Sons, Inc., New York, New York, U.S.A., pp. 233-269.

Deaton, A. S.: 1975, Models and Projections of Demand in Post-War Britain, Chapman and Hall, London, England.

Deaton, A., and J. Muellbauer: 1980a, Economics and Consumer Behavior, Cambridge University Press, Cambridge, England.

Deaton, A., and J. Muellbauer: 1980b, 'An Almost Ideal Demand System', American Economic Review, 70, 312-326.

Debreu, G.: 1958, 'Stochastic Choice and Cardinal Utility', Econometrica, 26, 440-444.

Duncan, G. M.: 1987, 'A Simple Approach to $M$-Estimation with Application to Two-Stage Estimators', Journal of Econometrics, 34, 373-389.

Geary, R. C.: 1949, 'A Note on "A Constant-Utility Index of the Cost of Living" ', Review of Economic Studies, 18, 65-66.

Hill, B. M.: 1963, 'The Three-Parameter Lognormal Distribution and Bayesian Analysis of a Point-Source Epidemic', Journal of the American Statistical Association, 58, 72-84.
Houthakker, H. S.: 1960, ‘Additive Preferences’, Econometrica, 28, 244-257.

Huber, P.: 1967, "The Behavior of Maximum Likelihood Estimates under Nonstandard Conditions', Proceedings of the Fifth Berkeley Symposium on Mathematical Statistics and Probability, 1, University of Califomia Press, Berkeley and Los Angeles, Califomia, U.S.A., pp. 221-233.

Johnson, N. L., and S. Kotz: 1970, Continuous Univariate Distributions-1, Houghton Mifflin Company, Boston, Massachusetts, U.S.A.

Klein, L. R., and H. Rubin: 1948, 'A Constant-Utility Index of the Cost of Living', Review of Economic Studies, 15, 84-87.

Lee, L. F., and M. M. Pitt: 1986a, 'Microeconometric Demand Systems with Binding Nonnegativity Constraints: The Dual Approach', Econometrica, 5, 1237-1242.

Lee, L. F., and M. M. Pitt: 1986b, 'Specification and Estimation of Consumer Demand Systems with Many Binding Non-Negativity Constraints', manuscript.

Lee, L. F., and M. M. Pitt: 1987, 'Microeconometric Models of Rationing, Imperfect Markets, and Non-Negativity Constraints', Journal of Econometrics, 36, 89-110.

Luce, R. D.: 1958, 'A Probabilistic Theory of Utility', Econometrica, 26, 193-224.

McFadden, D.: 1974, 'Conditional Logit Analysis of Qualitative Choice Behavior', in P. Zarembka (ed)., Frontiers in Econometrics, Academic Press, Inc., New York, New York, U.S.A.,pp. 105-142.

Malinvaud, E.: 1966, Statistical Methods of Econometrics, Rand McNally and Company, Chicago, Illinois, U.S.A.

May, K. O.: 1954, 'Transitivity, Utility, and Aggregation in Preference Pattems', Econometrica, 22, 1-14.

Mosteller, F., and P. Nogee: 1951, 'An Experimental Measure of Utility', Journal of Political Economy, 59, 371-404.

Papandreou, A. G., with the collaboration of O. H. Sauerlender, O. H. Brownlee, L. Hurwicz, and W. Franklin: 1957, 'A Test of a Stochastic Theory of Choice', University of California Publications in Economics, 16, 1-18.

Parks, R. W.: 1969, 'Systems of Demand Equations: An Empirical Comparison of Alternative Functional Forms', Econometrica, 37, 629-650.

Parks, R. W.: 1971, 'Maximum-Likelihood Estimation of the Linear Expenditure System' Journal of the American Statistical Association, 66, 900-903

Pollak, R. A.: 1970, 'Habit Formation and Dynamic Demand Functions', Journal of Political Economy, 78, 745-763.

Pollak, R. A., and T. J. Wales: 1969, 'Estimation of the Linear Expenditure System' Econometrica, 37, 611-628.

Quandt, R. E.: 1956, 'A Probabilistic Theory of Consumer Behavior', Quarterly Journal of Economics, 70, 507-536. 
Rao, C. R.: 1973, Linear Statistical Inference and Its Applications, John Wiley \& Sons, Inc., New York, New York, U.S.A.

Samuelson, P. A.: 1948, 'Some Implications of "Linearity" ', Review of Economic Studies, 15, 88-90.

Stone, R.: 1954, 'Linear Expenditure Systems and Demand Analysis: An Application to the Pattem of British Demand', Economic Journal, 64, 511-527.

Stone, R.: 1964, 'British Economic Balances in 1970: A Trial Run on Rocket', in P. E. Hart, G. Mills, and J. K. Whitaker (eds.), Econometric Analysis for National Economic Planning, Butterworths, London, England, pp. 65-95.

Stone, R., A. Brown, and D. A. Rowe: 1964, 'Demand Analysis and Projections for Britain: 1900-1970; A Study in Method', in J. Sandee (ed.), Europe's Future Consumption, 2, NorthHolland, Amsterdam, Holland, pp. 200-225.

Theil, H.: 1965. 'The Information Approach to Demand Analysis', Econometrica, 33, 67-87.

Wales, T. J., and A. D. Woodland: 1983, 'Estimation of Consumer Demand Systems with Binding Non-Negativity Constraints', Journal of Econometrics, 21, 263-285.

Woodland, A. D.: 1979, 'Stochastic Specification and the Estimation of Share Equations', Journal of Econometrics, 10, 361-383. 